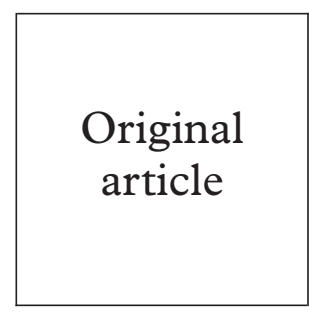

London School of Hygiene and Tropical Medicine, London, UK P Mayaud $\mathrm{H}$ A Weiss J Todd

H Grosskurth R J Hayes

D C W Mabey

African Medical and Research Foundation (AMREF), Mwanza, Tanzania

P Mayaud

G ka-Gina

H Grosskurth

Section of Infectious Diseases Department of Medicine, Imperial College School of

Medicine, London, UK

D K Gill

C J N Lacey

Regional Medical Office, Mwanza,

Tanzania

E Uledi*

L Kopwe

National Institute for Medical Research (NIMR), Mwanza, Tanzania

J Todd

*Died April 2000

Correspondence to: Philippe Mayaud, Department of Infectious and Tropical Diseases, London School of Hygiene and Tropical Medicine, Keppel Street, London WC1E 7HT, UK philippe.mayaud@1shtm.ac.uk

Accepted for publication 15 February 2001

\title{
The interrelation of HIV, cervical human papillomavirus, and neoplasia among antenatal clinic attenders in Tanzania
}

Philippe Mayaud, Dilbinder K Gill, Helen A Weiss, Evelyn Uledi, Lilian Kopwe, James Todd, Gina ka-Gina, Heiner Grosskurth, Richard J Hayes, David CW Mabey, Charles J N Lacey

Objectives: To determine the prevalence and interrelation of cervical human papillomavirus (HPV) genotypes, squamous intraepithelial lesions (SIL), HIV, and other reproductive tract infections (RTIs) among urban antenatal clinic attenders in Mwanza, Tanzania.

Methods: Genital swabs were collected from 660 pregnant women and tested for a range of RTIs and for cervical cytology. Cervical HPV-DNA was detected by PCR and genotyped. HIV and syphilis serologies were performed.

Results: HPV prevalence was 34\% (209/612 women). Of the 144 typeable samples, 83\% were high risk (HR-HPV) oncogenic strains (56\% HPV 16 related types). SIL was detected in 43 women $(7 \%)$, with high grade SIL in $3 \%$. There was a high prevalence of HIV $(15 \%)$, and of any RTI (83\%). Genital warts were detected in 20 women (3\%). HPV infection was associated with some behavioural factors (short duration of relationship, single status, not using condoms) and gonorrhoea. There was no overall association between HPV and HIV (OR=1.02, 95\% CI 0.6-1.6), but a non-significant trend towards a stronger association with HR-HPV in women aged $15-19(\mathrm{OR}=2.79,95 \% \mathrm{CI} 0.8-9.5)$ and women aged $\geqslant 30(\mathrm{OR}=3.20,95 \% \mathrm{CI} 0.7-15)$. SIL was associated with HPV (OR=3.66, 95\% CI 1.9-7.0), but not significantly with HIV (OR=1.54, 95\% CI 0.7-3.4). Prevalence of SIL was higher among women dually positive for HPV/HIV compared to HPV infection only $(21 \% v 12 \%)$, although this difference was not statistically significant $(\mathrm{p}=0.17)$.

Conclusions: HPV infection was highly prevalent in this young antenatal population. The association of HIV with HR-HPV types in older women may suggest that the principal HIV/HPV interaction in this population is for HIV to upregulate HPV persistence, leading to subsequent development of SIL.

(Sex Transm Inf 2001;77:248-254)

Keywords: human papillomavirus; squamous intraepithelial lesion; HIV/AIDS; Africa

\section{Introduction}

Cervical cancer is the most common female cancer in sub-Saharan Africa, and estimated age standardised incidence rates in eastern and southern Africa are among the highest in the world, being fourfold higher than those in North America and Europe. ${ }^{1}$ Certain human papillomavirus (HPV) genotypes, such as HPV types 16 and 18 , have been shown to be causative agents of cervical cancer. ${ }^{2}$ Human papillomaviruses causing genital infection fall into two phylogenetic families, known as "high risk" (HR) and "low risk" (LR) oncogenic HPVs, showing a greater and lesser association respectively with cervical cancer and precancer. $^{2}{ }^{3}$ High risk HPVs show an increasing gradient of frequency through the precursor lesions of cervical cancer, which are referred to as low grade and high grade squamous intraepithelial lesions (LSIL, HSIL). ${ }^{4}$

Both genital HPV and HIV are sexually transmitted infections (STIs) and may interact, but the nature of any such interaction has not been determined. Published data from Africa to date do not support an increased risk of cervical cancer in women with HIV infection, ${ }^{5-7}$ although data from America and Europe do. ${ }^{8}$
Furthermore, no study in Africa has systematically tested women for the full range of reproductive tract infections (RTIs), or examined any potential interaction between RTIs, HPV and neoplasia, and HIV. The high prevalence of HIV and cervical cancer and the known interactions between HIV and STIs in African populations gives an added urgency to the need for such data.

The objectives of this study were therefore to determine the prevalence, and study the interrelation, of cervical HPV, squamous intraepithelial lesions (SIL), HIV, and other RTIs, in an antenatal population in Tanzania.

\section{Materials and methods}

STUDY POPULATION AND SETTING

All pregnant women aged 15 years and over, seen at the largest urban antenatal clinic in Mwanza, Tanzania, over the period April to December 1994 were eligible for inclusion in a study seeking to evaluate a WHO risk assessment strategy for the detection of cervical infections. ${ }^{10}$ Every fifth woman was recruited to the study. Informed written consent was sought and the patient was seen by a female clinician in a private room. A standard questionnaire 
Table 1 Prevalence of HPV infection, cervical dysplasia, and other sexually transmitted or reproductive tract infections (STI/RTI) among antenatal clinic attenders in Mwanza, Tanzania

\begin{tabular}{lll}
\hline & No infected/total & \\
\hline HPV-DNA positive & $209 / 612$ & $34 \%$ \\
Identified types & $144 / 209$ & \\
High risk HPV-DNA types† & $120 / 144$ & \\
Low risk HPV-DNA types $\ddagger$ & $40 / 144$ & \\
$\quad$ Unidentified types & $65 / 209$ & \\
SILS & $43 / 607$ & $7.1 \%$ \\
Borderline dyskaryosis (ASCUS/LSIL) & $9 / 607$ & $1.4 \%$ \\
Mild dyskaryosis (LSIL) & $18 / 607$ & $3 \%$ \\
Moderate dyskaryosis (HSIL) & $12 / 607$ & $2 \%$ \\
Severe dyskaryosis (HSIL) & $4 / 607$ & $0.7 \%$ \\
HIV & $100 / 655$ & $15 \%$ \\
Other STI/RTI & $258 / 656$ & $39 \%$ \\
$\quad$ Candida spp & $138 / 660$ & $21 \%$ \\
Bacterial vaginosis & $108 / 656$ & $16 \%$ \\
Trichomonas vaginalis & $49 / 657$ & $7 \%$ \\
Neisseria gonorrhoeae and/or Chlamydia & & $8 \%$ \\
trachomatis & $55 / 659$ & $3 \%$ \\
Active syphilis (RPR+/TPHA+) $\|$ & $20 / 660$ & $83 \%$ \\
Genital warts & $533 / 645$ & \\
Any STI/RTI above & & \\
\hline
\end{tabular}

${ }^{\star}$ Denominators vary for HPV, SIL, HIV, and STI, as results are missing for some women. †HPV high risk group includes HPV types $16,18,26,31,33,35,39,45,51,52,55,56,58,59$, 68, $\mathrm{mm} 4, \mathrm{~mm} 7, \mathrm{~mm} 9$.

$\ddagger$ HPV low risk group includes HPV types $6,11,53,54,66, \mathrm{~mm} 8$.

§Note, both UK reporting criteria for dysplasia ${ }^{11}$ and equivalent categories in the Bethesda classification in parentheses ${ }^{12}$ are reported in this table.

ๆSIL = squamous intraepithelial lesions; LSIL = low grade SIL; HSIL = high grade SIL; ASCUS

$=$ atypical squamous cells of uncertain significance.

|| "Active syphilis": any dual positivity on RPR (whatever the titre) and TPHA.

elicited information on demographic characteristics, obstetric, contraceptive and sexual history, past STIs, and current genital tract symptoms. General, pelvic, and speculum examination and sample collection were performed. Depending on symptoms elicited or signs detected, patients were treated syndromically for their STIs according to the Tanzanian national guidelines. Patients were counselled on low risk sexual behaviour, provided with condoms if necessary, and given a follow up appointment after 7 days for their comprehensive laboratory results. At the follow up visit additional treatment was provided for infections that were not covered by syndromic treatment at the initial visit.

\section{SAMPLES TAKEN AND LABORATORY METHODS} Vaginal and cervical samples

After introduction of a clean, non-lubricated speculum, a first vaginal swab was used to prepare a wet saline preparation which was screened for the presence of typical motile trichomonads as evidence of $T$ vaginalis, and the presence of budding cells and/or pseudohyphae as evidence of infection with Candida spp. A second vaginal swab was used to prepare a Gram stained smear for the examination of vaginal flora type, identification of bacterial vaginosis, and presence of Candida spp (using the same criteria). Bacterial vaginosis was defined as the presence of clue cells constituting more than $20 \%$ of vaginal epithelial cells per high power field on the Gram stained smear. After cleaning of the cervix, two endocervical swabs were collected. One swab was immediately inoculated onto a modified Thayer-Martin agar plate for the diagnosis of Neisseria gonorrhoeae (NG) according to standard methods, while a second swab was preserved in transport medium for later diagnosis of Chlamydia trachomatis (CT) by an antigen capture enzyme immunoassay (IDEIA, Novo Nordisk Diagnostics, Cambridge, UK) and confirmatory antibody blocking assay.

\section{Cervical cytology}

Cervical material was taken with a wooden Ayre's spatula, smeared on a slide, immediately fixed with methyl alcohol, and allowed to air dry. The smears were sent to a reference cytopathology laboratory at St Mary's Hospital, London. Cervical cytology was carried out using standard techniques and reporting criteria in United Kingdom. ${ }^{11}$ Cytotechnicians were unaware of the patient's HPV, STI, and HIV status. We have expressed results using both the UK terminology and equivalent categories derived from the Bethesda classification. ${ }^{12}$ We use SIL to describe all combined nuclear cytological abnormalities, LSIL for borderline and mild dyskaryosis and HSIL for moderate and severe dyskaryosis.

\section{HPV PCR}

Material for HPV PCR testing was collected using an endocervical cytobrush, placed in phosphate buffered saline and stored at $-70^{\circ} \mathrm{C}$. Sample tubes were vortexed then centrifuged at $10000 \mathrm{~g}$ for 10 minutes to pellet cellular material. Cell pellets were resuspended in $200 \mu \mathrm{l}$ of proteinase $\mathrm{K}$ buffer $(50 \mathrm{mM} \mathrm{KCl}, 2.5 \mathrm{mM}$ $\mathrm{MgCl}_{2}, 10 \mathrm{mM}$ TRIS-HCl pH 8.3, 0.45\% (v/v) NP-40 0.45\% (v/v) Tween-20, and $60 \mu \mathrm{g} / \mathrm{ml}$ Proteinase $\mathrm{K}$ ) and incubated at $55^{\circ} \mathrm{C}$ overnight then at $95^{\circ} \mathrm{C}$ for 10 minutes. All PCRs were performed as described by Gravitt et $a l^{13}$ using Amplitaq gold to enable a "hot start" reducing non-specific amplification of irrelevant DNA species. The biotinylated primers MY09/MY11, HBB01, and GH20/PC04 were utilised to enable detection of positive PCR products using a reverse blot hybridisation assay. ${ }^{13}$ PCR reaction tubes were prepared in a clean PCR hood. Tubes were taken to a designated sample room where $10 \mu \mathrm{l}$ of patients' DNA were added. Tubes containing reaction mixture and sample were then taken to another laboratory for amplification.

The resulting PCR products were electrophoresed through a $2 \%$ agarose gel, stained with ethidium bromide, and photographed under ultraviolet light. Positive PCR products were then applied to the reverse blot typing assay (Roche Molecular Systems) to determine HPV genotype. As this is a novel test we confirmed the HPV typing results for HPV types $6,11,16,18$, and 31 using type specific PCR primers. ${ }^{14}$

\section{Serology}

A venous blood specimen was collected for serological testing. Diagnosis of serological syphilis was made using both the Treponema pallidum haemagglutination assay (TPHA, Fujirebio, Tokyo, Japan) and the rapid plasma reagin (RPR) test (VD-25, Murex Diagnostics, Dartford, UK), with any dually seroreactive sera considered to be "active syphilis."

HIV serology was performed following the WHO testing strategy, ${ }^{15}$ using two different enzyme linked immunosorbent assays (ELISA: Vironistika HIV MIXT Microelisa, Organon, 
Netherlands, and Wellcozyme HIV-1+2, Murex Diagnostics, Dartford, UK). Discordant results were resolved by western blot (HIV-1 WesternBlot, Epitope, Beaverton, OR, USA).

DATA ANALYSIS

Data were analysed using STATA 6 (Stata Corp, 1997). Univariate logistic regression was used to initially identify factors associated with HPV infection and SIL respectively, and odds ratios (ORs) were calculated with 95\% confidence intervals ( $95 \%$ CIs). Factors that were statistically significant in the univariate analyses or were thought to be important predictors based on previous knowledge (such as age and marital status) were included in multivariate analyses.

ETHICAL ISSUES

Ethical clearance was obtained from the Ministry of Health and the National Institute for Medical Research in Tanzania, the London School of Hygiene and Tropical Medicine in the United Kingdom, and all procedures complied with ethical guidelines of the World Health Organization who funded the study. Written informed consent was obtained from all participants. STI diagnosis and treatment were provided free of charge. Pre-HIV and post-HIV test counselling was provided to study participants who wished to know their HIV serostatus. The cervical cytology testing was performed in London several years later. It was not therefore possible to recontact women with abnormal Papanicolaou smear results.

\section{Results}

A total of 668 women were eligible for enrolment. Eight women were excluded from the study, either because they presented with

Table 2 Association of sociodemographic and behaviour factors with HPV infection among 612 antenatal clinic attenders with known HPV serostatus

\begin{tabular}{|c|c|c|c|c|c|}
\hline & $\begin{array}{l}\text { HPV infected/total } \\
(\%)(209 / 612)\end{array}$ & $\begin{array}{l}\text { Crude OR }(95 \% \\
\text { CI) }\end{array}$ & $p$ Value $^{\star}$ & $\begin{array}{l}\text { Adjusted ORt } \\
(95 \% \mathrm{CI})\end{array}$ & $p$ Value \\
\hline \multicolumn{6}{|l|}{ Age (years) } \\
\hline $15-19$ & $50 / 144(35 \%)$ & 1.00 & \multirow[t]{3}{*}{0.26} & 1.00 & \multirow[t]{3}{*}{0.25} \\
\hline $20-29$ & $138 / 388(36 \%)$ & $1.04(0.7-1.5)$ & & $1.44(0.9-2.2)$ & \\
\hline $30-44$ & $21 / 80(26 \%)$ & $0.67(0.4-1.2)$ & & $1.28(0.6-2.6)$ & \\
\hline \multicolumn{6}{|l|}{ Marital status } \\
\hline Married & $188 / 564(33 \%)$ & 1.00 & \multirow[t]{2}{*}{0.15} & 1.00 & \multirow[t]{2}{*}{0.05} \\
\hline Not married & $21 / 48(44 \%)$ & $1.56(0.9-2.8)$ & & $1.92(1.0-3.7)$ & \\
\hline \multicolumn{6}{|l|}{ Education } \\
\hline Low primary or less $(<$ std 4$)$ & $32 / 95(34 \%)$ & 1.00 & \multirow[t]{3}{*}{0.71} & \multirow[t]{3}{*}{ - } & \\
\hline High primary (std $4-7)$ & $157 / 450(35 \%)$ & $1.05(0.7-1.7)$ & & & \\
\hline Beyond primary & $20 / 67(30 \%)$ & $0.84(0.4-1.6)$ & & & \\
\hline \multicolumn{6}{|l|}{ Age (years) at first intercourse } \\
\hline $12-14$ & $37 / 105(35 \%)$ & 1.00 & \multirow[t]{3}{*}{0.94} & \multirow[t]{3}{*}{ - } & \\
\hline $15-18$ & $128 / 374(34 \%)$ & $0.96(0.6-1.5)$ & & & \\
\hline$\geqslant 19$ & $44 / 133(33 \%)$ & $0.91(0.5-1.6)$ & & & \\
\hline \multicolumn{6}{|l|}{ Number of pregnancies (gravidity) } \\
\hline 1 & $78 / 202(39 \%)$ & 1.00 & \multirow[t]{5}{*}{0.44} & \multirow[t]{5}{*}{ - } & \\
\hline 2 & $46 / 149(31 \%)$ & $0.71(0.5-1.1)$ & & & \\
\hline 3 & $29 / 87(33 \%)$ & $0.79(0.5-1.3)$ & & & \\
\hline 4 & $27 / 74(36 \%)$ & $0.91(0.5-1.6)$ & & & \\
\hline$\geqslant 5$ & $29 / 99(29 \%)$ & $0.66(0.4-1.1)$ & & & \\
\hline \multicolumn{6}{|c|}{ Reported number of partners in past year } \\
\hline 1 & $192 / 559(34 \%)$ & 1.00 & \multirow[t]{2}{*}{0.74} & - & \\
\hline$>1$ & $17 / 53(32 \%)$ & $0.90(0.5-1.6)$ & & & \\
\hline Duration with current partner & & & & & \\
\hline$\geqslant 5$ years & $48 / 192(25 \%)$ & 1.00 & 0.001 & 1.00 & 0.003 \\
\hline$<5$ years & $161 / 420(38 \%)$ & $1.86(1.3-2.7)$ & & $1.90(1.2-2.9)$ & \\
\hline Condom use & & & & & \\
\hline Never & $190 / 538(35 \%)$ & 1.00 & 0.10 & 1.00 & 0.03 \\
\hline Ever & $19 / 74(26 \%)$ & $0.63(0.4-1.1)$ & & $0.55(0.3-1.0)$ & \\
\hline Oral contraceptive use & & & & & \\
\hline Never & $179 / 525(34 \%)$ & 1.00 & 0.94 & - & \\
\hline Ever & $30 / 87(34 \%)$ & $1.02(0.6-1.6)$ & & & \\
\hline Residence of current partner & & & & & \\
\hline Same household & $183 / 550(33 \%)$ & 1.00 & 0.17 & - & \\
\hline Elsewhere & $26 / 62(42 \%)$ & $1.44(0.8-2.5)$ & & & \\
\hline N gonorrhoeae infection & & & & & \\
\hline No & $202 / 598(34 \%)$ & 1.00 & 0.05 & 1.00 & 0.004 \\
\hline Yes & $7 / 11(64 \%)$ & $3.43(1.0-12)$ & & $4.46(1.2-16.0)$ & \\
\hline$C$ trachomatis infection & & & & & \\
\hline No & $200 / 578(35 \%)$ & 1.00 & 0.32 & - & \\
\hline Yes & $9 / 34(26 \%)$ & $0.68(0.3-1.5)$ & & & \\
\hline$T$ vaginalis infection & & & & & \\
\hline No & $181 / 509(36 \%)$ & 1.00 & 0.05 & 1.00 & 0.009 \\
\hline Yes & $25 / 99(25 \%)$ & $0.61(0.4-1.0)$ & & $0.52(0.3-0.9)$ & \\
\hline Genital warts & & & & & \\
\hline No & $199 / 594(34 \%)$ & 1.00 & 0.06 & 1.00 & 0.04 \\
\hline Yes & $10 / 18(56 \%)$ & $2.48(1.0-6.4)$ & & $2.80(1.1-7.5)$ & \\
\hline TPHA (past syphilis) & & & & & \\
\hline Negative & $186 / 549(34 \%)$ & 1.00 & 0.61 & - & \\
\hline Positive & $23 / 62(37 \%)$ & $1.15(0.7-2.0)$ & & & \\
\hline TPHA/RPR (active syphilis) & & & & & \\
\hline Negative & $191 / 561(34 \%)$ & 1.00 & 0.78 & - & \\
\hline Positive (dual) & $18 / 50(36 \%)$ & $1.09(0.6-2.0)$ & & & \\
\hline HIV-1 infection & & & & & \\
\hline No & $173 / 511(34 \%)$ & 1.00 & 0.92 & - & \\
\hline Yes & $33 / 96(34 \%)$ & $1.02(0.6-1.6)$ & & & \\
\hline
\end{tabular}

*Overall $\mathrm{p}$ value for each factor.

†Adjusted for age, marital status, duration with current partner, condom use, $N$ gonorrhoeae infection, $T$ vaginalis infection, and genital warts. 


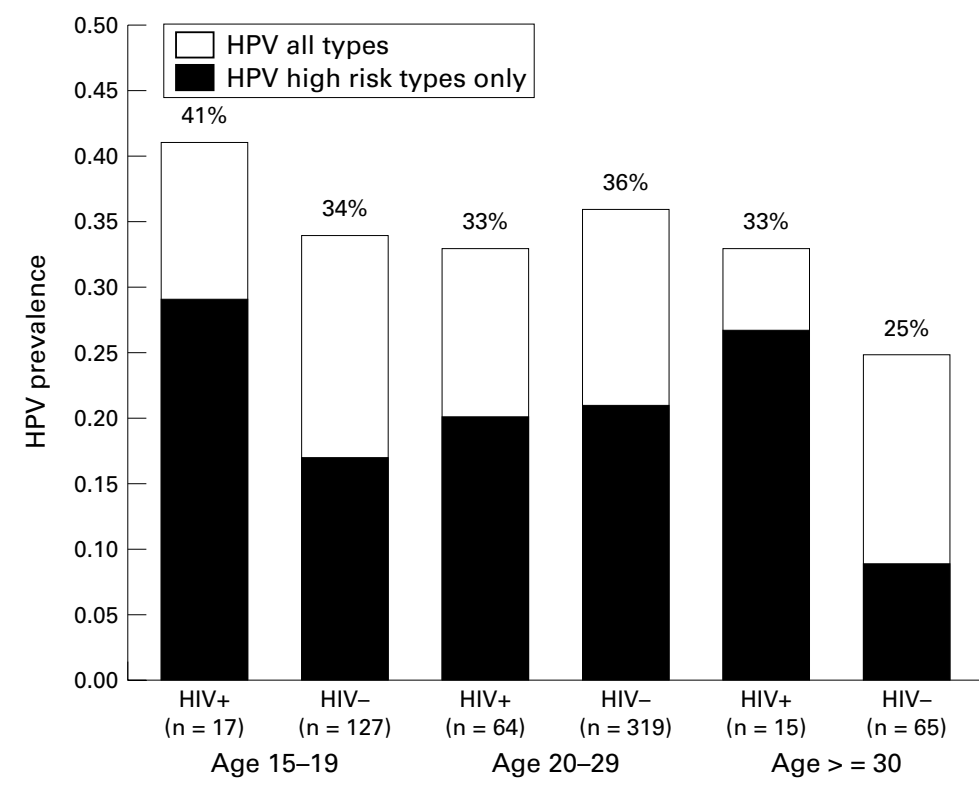

Figure 1 Prevalence of HPV infection by HIV and age among antenatal clinic attenders in Mwanza, Tanzania (607 women are included in this analysis, as HIV status was unavailable for five of the 612 women with known HPV status).

complications of their pregnancy (such as vaginal bleeding) and were immediately referred (five women), or because they refused permission for some or all of the study procedures (three women). Data were collected from the remaining 660 women. Details of the study population have been published previously. ${ }^{10}$ Briefly, the mean age of the study population was 23.4 (SD 5.1) years, and $24 \%$ were aged less than 20 . The majority of women $(92 \%)$ were currently married, of whom 84 (13\% of all women) were in polygamous unions. Ten per cent of women had reached secondary level education; $85 \%$ of women were housewives. Gravidity was on average 2.7.

Cervical samples from 48 women $(7 \%)$ were inadequate for PCR and excluded from PCR analyses. There were no significant differences in demographic, sexual behaviour, or biomedical variables between these women and those with adequate DNA samples. Cervical smears for $53(8 \%)$ women could not be interpreted, presumably because of heavy coexisting vaginal infections and were excluded from the SIL analysis. The prevalence of Trichomonas vaginalis was higher among women with inadequate cytology $(26 \%$ v 16\%, $\mathrm{p}=0.04)$.

HPV, SIL, AND HIV/STI

Cervical HPV infection was detected in $209 / 612$ women $(34 \%)$. Among these women, 24 genotypes were identified in 144 women $(69 \%)$, with 65 women $(31 \%)$ having cervical HPV infection not typeable by the 27 genotype reverse blot assay (table 1). Of the 144 women with identified genotypes, $120(83 \%)$ had at least one high risk type. The most common genotypes were HPV 16 (42 women), HPV 58 (26 women), mm7 (24 women), and HPV 33 (21 women), while only 14 women had HPV 18. Overall, the most common types belonged to the 16 phylogenetic group ( 81 women, $56 \%$ of those with known genotypes). Multiple HPV types were detected in $38 \%$ of women with an identified genotype.

SIL was detected in $43 / 607$ women $(7 \%)$, with 16 (3\% of all women) having HSIL and 27 (4\%) having LSIL (table 1). There was a high prevalence of HIV (15\%), vaginal, and cervical infections in this population. Genital warts were detected in 20 women (3\%), 10 of whom were HPV positive.

FACTORS ASSOCIATED WITH HPV INFECTION

There was little association of HPV infection with sociodemographic or sexual behaviour factors, including age, age at first sexual intercourse, and number of pregnancies (table 2). Multivariate analysis showed significant associations of HPV infection with the following factors: being unmarried $(\mathrm{OR}=1.92,95 \% \mathrm{CI}$ 1.0-3.7), duration of current relationship of less than 5 years $(\mathrm{OR}=1.90,95 \%$ CI $1.2-2.9)$, gonococcal infection $(\mathrm{OR}=4.46,95 \% \mathrm{CI}$ $1.2-16.0)$, and genital warts $(\mathrm{OR}=2.80,95 \%$ CI 1.1-7.5). HPV infection was inversely correlated with condom use $(\mathrm{OR}=0.55,95 \%$ CI $0.3-1.0)$ and with Trichomonas vaginalis $(\mathrm{OR}=0.52,95 \%$ CI $0.3-0.9)$. No factors were found to be significantly associated with HR-HPV infection (data not shown).

ASSOCIATION OF HPV AND HIV

There was no evidence of a direct association between HPV and HIV infection $(\mathrm{OR}=1.02$, 95\% CI 0.6-1.6) (table 2).

Analyses were then stratified by high risk/low risk HPV types, and adjusted for potential confounders (marital status, duration of time with partner, and condom use). There was no evidence of an association between HIV status and either high risk (HR) HPV type $(\mathrm{OR}=1.31,95 \%$ CI $0.8-2.2)$ or low risk (LR) types $(\mathrm{OR}=1.09,95 \%$ CI $0.4-3.3)$.

Prevalence of HPV infection by HIV status, stratified by age is shown in figure 1. Among HIV negative women, HPV prevalences for women aged $<20$ years, $20-29$ years, and $\geqslant 30$ years were $34 \%, 36 \%$, and $25 \%$ respectively. Among HIV positive women, HPV prevalences were $41 \%, 33 \%$, and $33 \%$.

There was some suggestion that associations between HIV and HPV, especially high risk types, varied with age. Associations between HIV and HR-HPV types were stronger among women aged 15-19 (OR=1.99, 95\% CI $0.6-6.2)$ and $30-44 \quad(\mathrm{OR}=3.58,95 \%$ CI 0.9-15) compared with women aged 20-29 $(\mathrm{OR}=1.09,0.9-1.3)$, although the difference by age group was not statistically significant $(\mathrm{p}=0.19)$

FACTORS ASSOCIATED WITH SIL

There was some suggestion of an increased risk of SIL with increasing age and among women with a partner not living in the same household (table 3). There were no other significant associations with any of the factors shown in table 2 .

After adjusting for age, SIL was significantly more prevalent among HPV positive women $(14 \%$ v $4 \%)$ especially among women with HR-HPV (17\%). SIL was also slightly more 
Table 3 Prevalence and associations of SIL with age, HPV, and HIV among 607 antenatal clinic attenders with cytology

\begin{tabular}{lll}
\hline & $\begin{array}{l}\text { SIL positive/ } \\
\text { total (\%) }\end{array}$ & OR $(95 \%$ CI)* \\
\hline Total & $43 / 607(7 \%)$ & \\
Age group (years) & & \\
$15-19$ & $7 / 148(5 \%)$ & 1.00 \\
$20-29$ & $28 / 381(7 \%)$ & $1.59(0.7-3.7)$ \\
$30-44$ & $8 / 78(10 \%)$ & $2.30(0.8-6.6)$ \\
Residence of current partner & \\
Same household & $35 / 548(6 \%)$ & 1.00 \\
$\quad$ Elsewhere & $8 / 59(14 \%)$ & $2.30(1.0-5.2)$ \\
HPV negative & $16 / 371(4 \%)$ & 1.00 \\
HPV positive & $26 / 190(14 \%)$ & $3.66(1.9-7.0)$ \\
High risk (HR) types & $18 / 107(17 \%)$ & $4.75(2.3-9.8)$ \\
Low risk (LR) types & $2 / 23(9 \%)$ & $2.17(0.5-10.1)$ \\
HIV negative & $33 / 513(6 \%)$ & 1.00 \\
HIV positive & $9 / 90(10 \%)$ & $1.54(0.7-3.4)$ \\
HPV- HIV- & $13 / 311(4 \%)$ & 1.00 \\
HPV- HIV+ & $3 / 59(5 \%)$ & $1.20(0.3-4.4)$ \\
HPV+ HIV- & $19 / 159(12 \%)$ & $3.24(1.6-6.8)$ \\
HPV+ HIV+ & $6 / 28(21 \%)$ & $6.16(2.1-17.9)$ \\
\hline
\end{tabular}

*Odds ratios for HPV and HIV are adjusted for age.

†Among the 607 women with cytology, HPV status was unknown for 46 women, and not typeable for 60 women.

†HIV status was unknown for four women with cytology.

prevalent in HIV positive women (10\% v 6\%) although this association was not statistically significant.

INTERRELATION OF HPV, HIV, AND SIL

Table 3 shows the association between SIL, HPV, and HIV. Women dually infected with HPV and HIV were at the highest risk $(\mathrm{OR}=6.16,95 \%$ CI 2.1-17.9) compared with women without either infection, although this risk was not significantly greater than the multiplicative effect of being HIV positive and HPV positive respectively ( $\mathrm{p}$ value for interaction $=0.55)$.

\section{Discussion}

We sought to determine the interrelation of HPV, HIV, and other STIs/RTIs in an African population. Any such associations which appear significant in a cross sectional study could result from biological interactions within individuals whereby one infection would increase the susceptibility to, or persistence of, another infection, or behavioural factors which might make coincident infection with certain STIs more likely.

Evidence is suggestive of such a potential interaction between HIV and HPV. Firstly, HIV could indirectly upregulate HPV replication through decreased generalised CD4 cell function ${ }^{16}$ and decreased Langerhans cell density $^{17}$ in the cervix. Secondly, HIV infection may directly upregulate HPV replication, as in vitro HIV-1 tat transactivates the HPV-16 upstream regulatory region, ${ }^{18}$ and in vivo tat mRNA levels have been shown to directly correlate with HPV 6/11 E7 mRNA. ${ }^{19}$

The most striking feature in our study is the lack of an overall significant association between HPV and HIV, which contrasts with results from other African studies. ${ }^{5}{ }^{70-28}$ This is unlikely to result from low power in our study, as the magnitude of the odds ratio was close to one (with an upper 95\% confidence limit of 1.6), and alternative explanations have to be sought. Some of the African studies suggesting an association were case-control studies conducted among high risk women such as commercial sex workers in Kenya ${ }^{20}$ Zaire, ${ }^{21}$ or Cote D'Ivoire ${ }^{23}{ }^{24}$ for whom exposure to HIV and multiple STI pathogens was very high, thereby increasing the chance to observe associations between sexually transmissible pathogens. Other African studies were conducted among older women than in Mwanza (median age 26 years in Malawi, ${ }^{26} 27$ in Abidjan, ${ }^{24}$ and 29 in Nairobi $^{27} v 23$ years in Mwanza) or in populations with much higher HIV prevalences ( $>40 \%$ in Malawi and Abidjan); thus it is likely that duration of HIV may have been longer in such women, with higher levels of immunosuppression and lower CD4 counts. Prospective studies of HPV and HIV have clearly demonstrated that women with HIV infection are much more likely than HIV negative women to have persistent $\mathrm{HPV}^{2629-31}$ and the effect is stronger in women of older ages and with lower CD 4 counts. ${ }^{26}{ }^{31}$ Analysis of our data by age showed stronger associations between HIV and HPV, particularly with high risk HPV strains, among the youngest and oldest age groups. Similar associations between HPV and HIV in young (under age 20) and older (above age 30) age groups were also found in a community based study in rural Uganda. ${ }^{28}$ The association between HPV and HIV among women aged under 20 is likely to be due to residual confounding through high risk sexual behaviour, or that of their partners. The association seen in older ages may be due to an effect of HIV on HPV persistence. However, these associations have to be interpreted with caution, as the interactions with age were not significant.

We suggest that the effect of HIV in upregulating HPV persistence with more likely progression to SIL may be obscured in the heterogeneity of the populations and their ages in the published African studies. This appears to be demonstrated in the range of associations reported between HIV and SIL where definite associations have been seen, ${ }^{7} 21242729$ equivocal associations have been demonstrated, ${ }^{22}{ }^{26}$ or no association found. ${ }^{52024}$ In our study, the prevalence of SIL was higher among HIV positive women, but the association did not reach statistical significance. Given the high HIV prevalence in many parts of Africa, the extent to which HIV modifies persistence of HPV and thus development of SIL and cervical cancer needs further assessment. A population based study of cervical cancer in Uganda, a country which has been one of the earliest and hardest hit by the HIV epidemic in Africa, has shown a doubling of incidence of cervical neoplasia between 1954-60, 1968-70, and 1989-91, but a stabilisation in the 1990 s with no indication that the disease would present at earlier stages, while an increasing incidence of other AIDS associated cancers, such as Kaposi's sarcoma (KS) and squamous cell carcinoma of the conjunctiva (SCCC) continued throughout the $1990 \mathrm{~s}^{32}$ Similar findings were reported from a cancer registry study in Zimbabwe, with no apparent increase of cervical cancer through the 1990s, while rates of AIDS associated KS 
and SCCC soared. ${ }^{33}$ These observations suggest that the prediction of maturing HIV epidemics promoting secondary epidemics of HPV, resulting in turn in severe epidemics of cervical cancer, ${ }^{21} 2728$ may not be founded yet. Although this does not leave room for complacency, longitudinal cohort, or better, intervention studies will be required to disentangle the complex relation between HIV and HPV.

There have been few studies which have collected information on HPV, HIV, and SIL in general populations from sub-Saharan Africa, as most have been conducted in sex workers ${ }^{20-24}$ women attending a national cervical cancer referral centre in Tanzania, ${ }^{5}$ gynaecology outpatients in Abidjan, ${ }^{7} \mathrm{HIV} /$ infectious diseases outpatients in Dakar, ${ }^{28}$ and an antenatal cohort deliberately chosen to have a majority of HIV infected women also in Abidjan. ${ }^{23}{ }^{24}$ Studies of less selected populations include research on pregnant women enrolled at delivery in Malawi, ${ }^{26}$ family planning clinic attenders in Nairobi, ${ }^{27}$ and our own study. To date, there has been only one population based study of HPV and HIV conducted in rural Uganda, ${ }^{28}$ but it was based on self collected vaginal swabs with no previous validation against cervical sampling and no cervical cytology data. Thus, our study contributes useful information on the prevalence of HPV, SIL and associated risk factors in a more general population. Only our study and two others $^{734}$ have used a methodology capable of distinguishing multiple genotypes.

The HPV prevalence in the present study was $34 \%$, with over $80 \%$ of identified strains belonging to oncogenic types, and very frequent infection multiple types (38\%). The prevalence found in Mwanza is higher than in most comparable studies ${ }^{26}{ }^{27}$ and higher than the prevalence found in the population based study of rural Uganda $(16 \%) .{ }^{28}$ The subjects in our study had the lowest median age of any of the previously quoted studies, and the strongest determinants of HPV prevalence have been shown to be young age and markers of sexual behaviour, such as age at first intercourse, lifetime number of partners, and duration of relationship. $^{35}$

We identified a number of risk factors for HPV infection. In contrast with previous studies, there was little association with sexual behaviour history, although this may not be entirely surprising in a population of predominantly married pregnant women, reporting little risky sexual behaviour. Differences in cultural and social factors may explain the difference noted with a population of family planning attenders in Nairobi for whom sexual behaviour factors were strongly associated with $\mathrm{HPV}^{27}$ An indirect marker of recent sexual behaviour, such as duration of current partnership, was, however, significantly associated with increased risk of HPV. This was also demonstrated in a previous report, ${ }^{35}$ where this variable was associated with the male partner's sexual behaviour. We did not find a positive association between HPV infection and markers of past or recent STI, except for gonococcal infection which was strongly associated with
HPV (OR=4.46, $\mathrm{p}=0.004)$. One could speculate that this association reflects a common link with recent exposure to sexual partners with an increased risk of infection.

The apparent protective effect of $T$ vaginalis for HPV infection was unexpected. It is possible that this is a measurement artefact, as $T$ vaginalis may affect the sensitivity of the HPV PCR through the severe inflammatory process it elicits, as was found in another study. ${ }^{26}$ This hypothesis is supported by the higher prevalence of $T$ vaginalis among women whose smears were not interpretable.

The public health importance of HPV arises from its association with cervical neoplasia, with persistent HPV infection being the causative factor of progression to SIL. ${ }^{35}$ The prevalence of SIL in this study (7\%) was similar to that in previous studies of African populations, in which estimates ranged from $6 \%$ to $12 \% .^{7234}$ The association between HPV and SIL found in our study also accords with previous studies conducted in Africa. ${ }^{726} 2729$

The high prevalence of STIs, HIV, HPV infection, and SIL among this apparently "low risk" population is a cause for great concern. While the control of bacterial STIs has been given renewed emphasis for its potential preventive effect on HIV transmission, control of viral STIs will remain problematic since diagnostic and therapeutic tools are lacking. Thus, prevention will remain of paramount importance. Unfortunately, little has been done in Africa to raise awareness about the risk of genital cancers and sexually transmissible HPV. Moreover, screening of either HPV and/or its early consequences (SIL) in settings where women seek health care, such as family planning, antenatal, and mother and child clinics will be riddled with logistical problems and will be too expensive to run in most developing countries. ${ }^{36}$ The greatest hopes remain with the development of therapeutic or prophylactic vaccines, to protect already infected women from progressing to cancer, or to prevent acquisition before onset of sexual activity, respectively. ${ }^{37}$ Careful community based studies will be required to provide further information on the epidemiology of HPV in Africa, particularly in adolescent populations, and to assess the acceptability and feasibility of vaccination. The authors thank the Tanzanian National AIDS Control Pro-
gramme and the Tanzanian National Medical Research Coordinating Committee for permission to undertake this study, the Ministry of Health authorities at national and regional levels for Ministry of Health authorities at national and regional levels for
their support, the staff of the Makongoro clinic for patients their support, the staff of the Makongoro clinic for patients
recruitment, the Microbiology Department of the National Institute for Medical Research in Mwanza for STD laboratory work, the Serology Department of the Bugando Medical Centre for HIV testing, Professor DV Coleman and staff of the Cytology Department at St Mary's Hospital London, for performing cervical cytology, and Roche Molecular Diagnostics, as well as Patti Gravitt and Ray Apple, for donation of the reverse blot typing strips and technical assistance. Other key staff who participated in parts of the study include Domitilia Manoko, Medard Rwakatare, Janet Marealle, and Jan Cornelissen, who are warmly thanked. Finally, the authors are immensely grateful to all the women participating in the study.

The study was supported by the STD Department of the Global Programme on AIDS at the World Health Oranization Global Programme on AIDS at the World Health Organization (WHO/GPA/STD), Geneva, Switzerland. We are grateful to Dr Piot (currently Head of UNAIDS, formerly with WHO/GPA/ STD) and to Professor Laga (Institute of Tropical Medicine, Antwerp, Belgium) for their support in setting up this study. The Mwanza STD/HIV Intervention and Research programme
was supported by other sponsors, including the Commission of 
the European Communities (DG VIII and DG XII), the former Overseas Development Administration (ODA) of the British Development, DFID), and he Centre for opment (CIM), Germany. HA Weiss is funded by the Medica Research Council, UK. CJN Lacey and DK Gill acknowledge the support of Cantab Pharmaceuticals Research Limited.

Contributors: PM, HG, RJH, and DCWM designed the study and PM implemented it with the participation of EU, LK, and Gka-G for the clinical component; JT oversaw data management; DKG carried out the HPV-DNA analysis, under the supervision of CJNL; HAW performed statistical analysis and in collaboration with other co-authors interpreted the results; PM, HAW, and CJNL wrote the first draft of the manuscript, which was commented on and revised by all co-authors.

Conflict of interest: None declared.

1 Parkin DM, Pisani P, Ferlay J. Estimates of the worldwide incidence of 25 major cancers in 1990. Int $\mathcal{f}$ Cancer 1999;80:827-41.

2 Walboomers JMM, Jacobs MV, Manos MM, et al. Human papillomavirus is a necessary cause of invasive cervical cancer worldwide. I Pathol 1999; 189:12-19

3 Van Ranst M, Kaplan JB, Burk RD. Phylogenetic classifiVan Ranst M, Kaplan JB, Burk RD. Phylogenetic classifimanifestations. F Gen Virol 1992;73:2653-60.

4 Lorincz AT, Reid R, Jenson AB, et al. Human papillomavirorincz AT, Reid R, Jenson AB, et al. Human papillomavirus infection of the cervix: relative risk associations of 15
common anogenital types. Obstet Gynecol 1992;79:328-37.

5 Ter Meulen J, Eberhardt HC, Luande J, et al. Human papillomavirus infection, HIV infection and cervical cancer in Tanzania, east Africa. Int $\mathcal{F}$ Cancer 1992;51:515-21.

6 Newton R, Grulich A, Beral V, et al. Cancer and HIV infection in Rwanda. Lancet 1995;345:1378-9.

$7 \mathrm{La}$ Ruche G, You B, Mensah-Ado I, et al. Human papillomavirus and human immunodeficiency virus African women. Int $\mathcal{f}$ Cancer 1998;76:480-6.

8 Goedert JJ, Cote TR, Virgo P, et al. Spectrum of AIDS-associated malignant disorders. Lancet 1998;351: 1833-9.

9 Serriano D, Carrieri P, Pradier C, et al. Risk of invasive cervical cancer among women with, or at risk for HIV infection. Int 7 Cancer 1999;82:334-7.

10 Mayaud P, Uledi E, Cornelissen J, et al. Risk scores to detect cervical infections in urban antenatal clinic attenders in Mwanza, Tanzania. Sex Transm Inf 1998;74:S139-46.

11 Evans DMD, Hudson EA, Brown CL, et al. Terminology in gynaecological cytopathology: report of The Working Party of The British Society for Clinical Cytology. 7 Clin Pathol 1986;39:933-44

12 Richart RM. A modified terminology for cervical intraepithelial neoplasia. Obstet Gynecol 1990;75:131-3.

13 Gravitt PE, Peyton CL, Apple RJ, et al. Genotyping of 27 human papillomavirus types by using L1 consensus PCR products by a single-hybridization, reverse line blot detection method. F Clin Microbiol 1998;36:3020-7.

14 Van den Brule AJC, Meijer CJ, Bakels V, et al. Rapid detection of human papillomavirus in cervical scrapes by combined general primer-mediated and type-specific polymerase chain reaction. F Clin Microbiol 1990;28:2739polym.

15 UNAIDS and WHO. Revised recommendations for the selection and use of HIV antibody tests. Weekly Epidemioselection and use of HIV ant
logical Record 1997;12:81-8.

16 Laurence J, Friedman SM, Chartash EK, et al. Human immunodeficiency virus infection of helper $\mathrm{T}$ cell clones Early proliferative defects despite intact antigen-specific recognition and interleukin 4 secretion. F Clin Invest 1989; 83:1843-8

17 Spinillo A, Tenti P, Zappatore R, et al. Langerhans cell counts and cervical intraepithelial neoplasia in women with human immunodeficiency virus infection. Gynecol Oncol 1993;48:210-13.
18 Tornesello ML, Buonaguoro FM, Beth-Giraldo E, et al. Human immunodeficiency virus type 1 tat gene enhances human papillomavirus early gene expression. Intervirology 1993;36:57-64.

19 Arany I, Evans T, Tyring SK. Tissue specific HPV expression and downregulation of local immune responses
in condylomas from HIV seropositive individuals. Sex Transm Inf 1998;74:349-53.

20 Kreiss JK, Kiviat NB, Plummer FA, et al. Human immunodeficiency virus, human papillomavirus and cervical intraepithelial neoplasia in Nairobi prostitutes. Sex Transm Dis 1992;19:54-9.

21 Laga M, Icenogle JP, Marsella R, et al. Genital papillomavirus infection and cervical dysplasia - opportunistic complications of HIV infection. Int $\mathcal{F}$ Cancer 1992;50:45-8.

22 Langley CL, Benga-De E, Critchlow CW, et al. HIV-1, HIV-2, human papillomavirus infection and cervical neoplasia in high-risk African women. AIDS 1996;10:41317.

23 Piper MA, Severin ST, Wiktor SZ, et al. Association of human papillomavirus with HIV infection and CD4 cell count in women with high or low numbers of sex partners. Sex Transm Inf 1999;75:253-7.

24 Vernon SD, Unger ER, Piper MA, et al. HIV and human papillomavirus as independent risk factors for cervical neoplasia in women with high or low numbers of sex partners. Sex Transm Inf 1999;75:258-60.

25 Coll-Seck A, Awa-Faye M, Critchlow CW, et al. Cervical intraepithelial neoplasia and human papillomavirus infection among Senegalese women seropositive for HIV-1 or HIV-2 or seronegative for HIV. Int $\mathcal{f} S T D$ AIDS 1994;5:189-93.

26 Miotti PG, Dallabetta GA, Daniel RW, et al. Cervical abnormalities, human papillomavirus and human immunodeficiency virus infections in women in Malawi. $\mathcal{F}$ Infect Dis 1996;173:714-17.

27 Temmerman M, Tyndall MW, Kidula N, et al. Risk factors

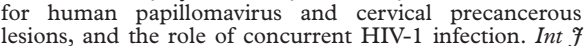
Gynaecol Obstet 1999;65:171-81.

28 Serwadda D, Wawer MJ, Shah KV, et al. Use of hybrid capture assay of self-collected vaginal swabs in rural Uganda for detection of human papillomavirus. F Infect Dis 1999;180:1316-9.

29 La Ruche G, Leroy V, Mensah-Ado I, et al. Short-term follow-up of cervical squamous intraepithelial lesions associated with HIV and human papillomavirus infections in Africa. Int F STD AIDS 1999;10:363-8.

30 Vernon SD, Reeves WC, Clancy KA, et al. A longitudinal study of human papillomavirus DNA detection in human immunodeficiency virus type 1 seropositive and seronegative women. F Infect Dis 1994, 169:1108-12.

31 Sun X-W, Kuhn L, Ellerbrock TV, et al. Human papillomavirus infection in women infected with the human immunodeficiency virus. N Engl f Med 1997;337:1343-9.

32 Parkin DM, Wabinga $\mathrm{H}$, Nambooze S, et al. AIDS-related cancers in Africa: maturation of the epidemic in Uganda. AIDS 1999;13:2563-70

33 Chokunonga E, Levy LM, Bassett MT, et al. AIDS and cancer in Africa: the evolving epidemic in Zimbabwe. AIDS 1999, 13:2383-8.

34 Chabaud M, Le Cann P, Mayelo V, et al. Detection by PCR of human papillomavirus genotypes in cervical lesions of Senegalese women. $7 \mathrm{Med}$ Virol 1996;49:259-63.

35 Burk RD, Ho GYF, Beardsley L, et al. Sexual behaviour and partner characteristics are the predominant risk factors for genital human papillomavirus infection in young women. $\mathcal{F}$ Infect Dis 1996;1174:679-89.

36 Bishop A, Wells E, Sherris J, et al. Cervical cancer: evolving prevention strategies for developing countries. Reproductive Health Matters 1995;6:60-71.

37 Galloway DA. Is vaccination against human papillomavirus a possibility? Lancet 1998;51(suppl III):SIII22-4. 